Journal of Elasticity 18: 289 (1987)

(C) Martinus Nijhoff Publishers, Dordrecht - Printed in the Netherlands

Erratum

\title{
Nonlinear surface acoustic waves on an elastic solid of general anisotropy
}

\section{R.W. LARDNER}

Department of Mathematical Sciences, King Fahd University of Petroleum \& Minerals, Dhahran, Saudi Arabia

Journal of Elasticity 16 (1986) pp. 63-73

Equations (55) and (56) should have been printed as follows.

For $0<k^{\prime}<k$,

$$
\begin{aligned}
H\left(k, k^{\prime}\right)= & \frac{P^{\prime}}{Q}+\frac{2}{Q k} \sum_{l \neq n} \beta_{i}^{\prime} \beta_{i} \beta_{n}\left(k-k^{\prime}\right) \\
& \times\left\{c_{i 2 j 2} \frac{s^{(l)} k^{\prime}+s^{(n)}\left(k-k^{\prime}\right)}{k}+c_{i 2 j 1}\right\} \\
& \times N_{m l n}^{(1)} L_{j m}^{-1}\left(\frac{s^{(l)} k^{\prime}+s^{(n)}\left(k-k^{\prime}\right)}{k}\right) .
\end{aligned}
$$

For $k^{\prime}>k$,

$$
\begin{aligned}
H\left(k, k^{\prime}\right)= & \frac{Q^{\prime}}{Q}+\frac{2}{Q k} \sum_{l, n} \beta_{i}^{\prime} \beta_{i} \bar{\beta}_{n}\left\{c_{i 2 j 2} \frac{s^{(l)} k^{\prime}+\overline{s^{(n)}}\left(k-k^{\prime}\right)}{k}+c_{i 2 j 1}\right\} \\
& \times\left\{k^{\prime} N_{m n l}^{(2)}+\left(k-k^{\prime}\right) N_{m l n}^{(3)}\right\} L_{j m}^{-1}\left(\frac{s^{(l)} k^{\prime}+\overline{s^{(n)}}\left(k-k^{\prime}\right)}{k}\right) .
\end{aligned}
$$

The correct expressions were used in the subsequent paper by Lardner and Tupholme (J. Elasticity 16 (1986) pp. 251-65). 